\title{
Images in Cardiovascular Medicine: Tuberculous Aortitis
}

\author{
Mei-Mei Lin $^{1}$ and Hao-Min Cheng ${ }^{1-3}$
}

\begin{abstract}
Tuberculous aortitis is a rare disease entity indicative of disseminated tuberculosis. We report a case who presented with newly diagnosed hypertension one year after her last admission for pulmonary and pericardial tuberculosis. Chest CT and MRA, performed due to marked differences in pulse amplitude between upper and lower limbs, demonstrated long and severe segmental stenosis of the thoracic aorta. After the operation of bypass graft for the thoracic aorta, the patient recovered uneventfully. Tuberculous aortitis should be included in the list of differential diagnosis for secondary hypertension, especially if the patient has a recent disease history of pulmonary tuberculosis.
\end{abstract}

Key words: tuberculosis aortitis, secondary hypertension

(Intern Med 51: 1983-1985, 2012)

(DOI: 10.2169/internalmedicine.51.7690)

\section{Introduction}

Tuberculous aortitis (TA) is a rare entity that is invariably indicative of disseminated tuberculosis. TA is associated with aneurysm formation in about half of the cases with a possible complication of free aortic wall perforation. It involves abdominal and thoracic aorta with equal frequency, followed by ascending aorta and aortic arch. In rare circumstances, the stenotic segment of the involved aorta can present with marked pressure gradient between upper and lower limbs. Fatal outcomes are frequently reported even after anti-tuberculosis chemotherapy and surgical intervention.

\section{Case Report}

A 25-year-old woman had a past history of pulmonary tuberculosis (Fig. 1A, B) and tuberculous pericarditis diagnosed by positive sputum and pericardial fluid culture of Mycobacterium tuberculosis. Additional laboratory results showed leucocytes $17 \cdot 6 \times 10^{9} / \mathrm{L}$; hemoglobin $86 \mathrm{~g} / \mathrm{L}$; Creactive protein $31 \mathrm{mg} / \mathrm{dL}$; BUN $13 \mathrm{mg} / \mathrm{dL}$; creatinine 0.8 $\mathrm{mg} / \mathrm{dL}$; GOT $12 \mathrm{U} / \mathrm{L}$; GPT $17 \mathrm{U} / \mathrm{L}$. She had then completed a 10-month course of standard anti-tuberculosis regimen.

Two months after completion of the treatment, she was admitted to our ward with the presentation of new-onset hy- pertension. The following investigations were normal on admission: leucocytes $5.2 \times 10^{9} / \mathrm{L}$, hemoglobin $11 \mathrm{~g} / \mathrm{L} ; \mathrm{C}$ reactive protein $0.3 \mathrm{mg} / \mathrm{dL}$; BUN $11 \mathrm{mg} / \mathrm{dL}$; creatinine 0.7 $\mathrm{mg} / \mathrm{dL}$; GOT $14 \mathrm{U} / \mathrm{L}$; GPT $20 \mathrm{U} / \mathrm{L}$. The pleural effusion and pulmonary consolidation were completely resolved on follow-up chest X-ray and there was no fever, fatigue, or elevated CRP. Headache, probably related to new-onset high blood pressure, was noted and improved after antihypertensive treatment. In response to the new onset hypertension, we had performed a series of exams. The survey of secondary hypertension including rennin-angiotensionaldosterone profiles, serum concentration of catecholamine, and cortisol were essentially normal.

A marked difference in the pulse amplitude between upper and lower extremities was noted. Computed tomography (CT) and magnetic resonance angiography (MRA) of the chest showed a thickened pericardium with $3.9 \mathrm{~mm}$ in thickness (Fig. 1C, arrowhead) and a long, severe, segmental stenosis of the thoracic aorta (Fig. 1C, 1D, arrow; Fig. 2), which had been surrounded by the infected tissue of pulmonary and pericardial tuberculosis (Fig. 1A, B). A pressure gradient of about $100 \mathrm{mmHg}$ was measured by Doppler echocardiography. As a result of previous tuberculosis history, evidence of extrapulmonary tuberculosis had been sought comprehensively. No palpable lymph node was found through detailed physical examination. Computed tomogra-

${ }^{1}$ Division of Cardiology, Department of Medicine, Taipei Veterans General Hospital, Taiwan, ${ }^{2}$ Department of Medical Research and Education, Taipei Veterans General Hospital, Taiwan and ${ }^{3}$ National Yang-Ming University, School of Medicine, Taiwan

Received for publication March 9, 2012; Accepted for publication May 1, 2012

Correspondence to Dr. Hao-Min Cheng, hmcheng@vghtpe.gov.tw 

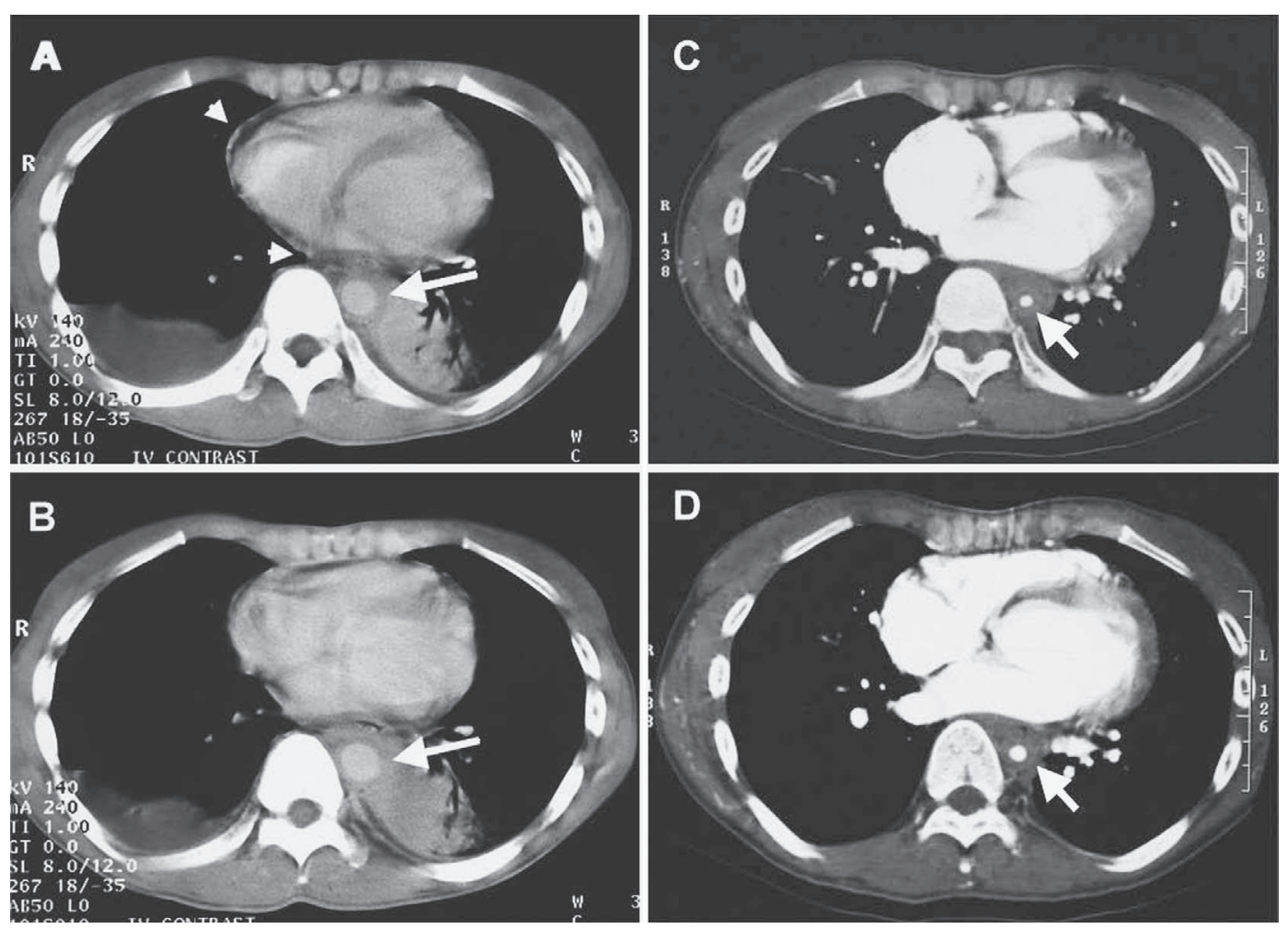

Figure 1. (A)(B) Computed tomography of the chest disclosed pulmonary consolidation in the superior segments of both lower lobes, which surrounded descending thoracic aorta with normal diameter (arrow). (C)(D): Computed tomography of the chest 12 months after last exam showed stenotic descending aorta which was around the same level of aorta surrounded by the previously infected segment (arrow).
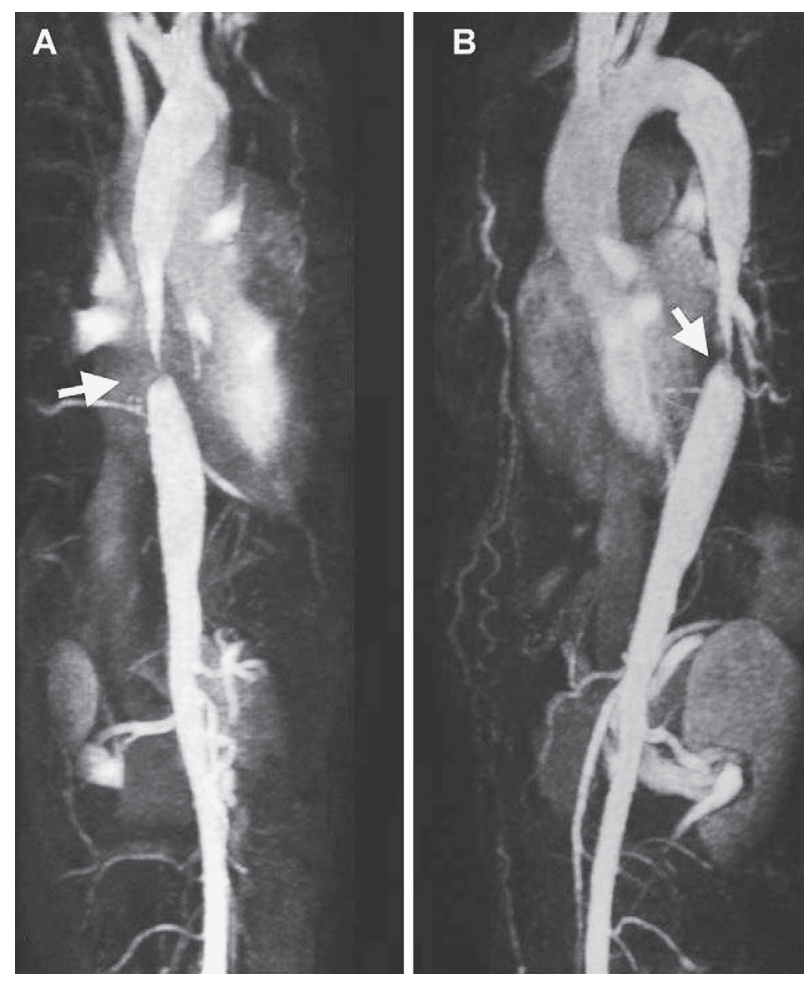

Figure 2. (A)(B): Magnetic resonance angiography (MRA) of the thoracic aorta disclosed long, severe, and segmental stenosis of the thoracic aorta (arrow). phy excluded the possibility of miliary tuberculosis or intraabdominal involvement. Magnetic resonance imaging also showed negative evidence of bone or joint tuberculosis. In addition, no focal neurologic signs or meningismus have ever been noted during the whole course of illness, which rendered the diagnosis of central nervous system tuberculosis very unlikely. Intraaortic vegetation is an unusual complication of infective endocarditis and could present with similar clinical pictures. However, no evidence of infective endocarditis was found on echocardiography.

Surgical intervention for the segmental stenosis of the thoracic aorta with bypass graft was performed under the impression of TA. During the operation, severe adhesion of pleural and lung tissue to the stenotic segment of aorta were found, which precluded biopsy because of the concern for safety. The patient recovered uneventfully. Two years after the bypass grafting to the aorta, all anti-hypertensive medication had been discontinued and routine check-up indicated her blood pressure was within normal range. Essentially normal chest film and echocardiography on follow-up suggested no recurrence of pulmonary and pericardial tuberculosis.

\section{Discussion}

TA, a rare disease entity involving the aorta, can be caused by direct extension from adjacent tuberculous tissue or by blood-borne seeding from a distant focus via the vasa 
vasorum, resulting in a stenosis and is invariably indicative of disseminated tuberculosis. More commonly, TA is associated with aneurysm formation in about half of cases with a possible complication of perforation of aorta $(1,2)$. It involves abdominal and thoracic aorta with equal frequency. Fatal outcomes are frequently reported even after antituberculosis chemotherapy and surgical intervention. Hypertension is the most common presentation (3), and is usually a consequence of renal artery stenosis or aortic coarctation. Mycotic aneurysm secondary to tuberculous infection of the aorta is another kind of TA, featuring presentation of pain, palpable mass, or hypovolemia secondary to leak, which is usually a consequence of the aneurysm $(1,4)$.

Pulmonary tuberculosis usually is localized to the apical and posterior segments of the upper lobes and superior segments of the lower lobe, and the latter is usually close to the descending thoracic aorta. The close spatial relationship between the stenotic thoracic aortic and the previously infected segment of pulmonary tuberculosis was clearly demonstrated in the present case. It suggested that direct extension was the most likely mechanism of disease progression of TA in this case, which indicates the need to improve our understanding of the course of tuberculous infection, especially when the initial infected foci are close to vital organs. We have presented this case also to remind physicians that TA should be included in the list of differential diagnoses for secondary hypertension, especially if the patient has a recent disease history of pulmonary tuberculosis.

The authors state that they have no Conflict of Interest (COI).

\section{References}

1. Kontogiannis V, Dalziel KL, Powell RJ. Papulonecrotic tuberculide and stenosis of the abdominal aorta. Rheumatology (Oxford, England) 39: 205-208, 2000.

2. Bukhary ZA, Alrajhi AA. Tuberculous aortitis. Ann Saudi Med 26: 56-58, 2006.

3. Gajaraj A, Victor S. Tuberculous aortoarteritis. Clin Radiol 32: 461-466, 1981

4. Gornik HL, Creager MA. Aortitis. Circulation 117: 3039-3051, 2008.

(C) 2012 The Japanese Society of Internal Medicine http://www.naika.or.jp/imindex.html 